\title{
Comparing the acute effect of myofascial release techniques and passive stretching on hind foot dynamic loading in patients with multiple sclerosis: A cohort-blinded study
}

\author{
Ayla Fil Balkan², Hilal Keklicek ${ }^{1}$, Yeliz Salci², Umut Altinkaynak ${ }^{3}$, Kadriye Armutlu² \\ 1 Department of Physiotherapy and Rehabilitation, Faculty of Health Sciences, Trakya University, Edirne, Turkey; \\ 2 Department of Physiotherapy and Rehabilitation, Faculty of Health Sciences, Hacettepe University, Ankara, Turkey; \\ ${ }^{3}$ Gercek Prosthetics and Orthotics Research and Development Group, Ankara, Turkey
}

\section{Summary}

Study aim: It is important for therapists to incorporate new practical methods into therapy programs when they have demonstrable efficacy in the treatment of multiple sclerosis. Investigating the acute effects of myofascial release techniques (MFR) and passive stretching (PS) on hind foot loading and the severity of spasticity in individuals with multiple sclerosis (MS) was the aim of the study.

Material and methods: Following the initial evaluation, 10 participants with MS ( $\mathrm{n}=20$ feet) were given MFR for the plantar flexor muscle group. After the day following the first visit, participants were asked to come again and PS was applied to the plantar flexor muscle groups after the evaluation. The severity of spasticity was assessed with the Modified Ashworth Scale (MAS). Dynamic loading parameters of the hind foot - medial and lateral maximum pressure $\left(\mathrm{N} / \mathrm{cm}^{2}\right)$, active contact areas $\left(\mathrm{cm}^{2}\right)$, contact percentiles $(\%)$ - were evaluated with dynamic pedobarography. Participants of the study were evaluated four times: (1) at the initial evaluation, (2) after MFR application, (3) 24 hours after the initial evaluation (pre-PS), (4) after PS.

Results: There were no differences in MAS ( $\mathrm{p}>0.05)$ according to time-dependent analyses $(\mathrm{p}>0.05)$. After MFR, the maximum pressure of the medial heel and active contact area were increased $(p<0.05)$ and there was a carryover effect on the maximum pressure of the right foot.

Conclusions: This study showed that MFR was an effective method for management of plantar flexor spasticity in patients with multiple sclerosis in the short term and there was a carryover effect in favor of MFR. There was no additional effect of PS.

Keywords: Multiple sclerosis - Musculoskeletal manipulations - Plantar pressure distribution - Gait

\section{Introduction}

Multiple sclerosis (MS) is a chronic inflammatory demyelinating disease of the central nervous system. MS leads to occurrence of focal lesions in the gray and white matter and to diffuse neurodegeneration in the entire brain and spinal cord [11]. The typical clinical features of MS are episodes of neurological disability [11, 27]. The relapsing and remitting episodes of neurological disability in MS reflect this pathological hallmark $[2,11]$. The pathological process of MS includes the breakdown of the blood-brain barrier, multifocal inflammation, demyelination, oligodendrocyte loss, reactive gliosis, and axonal degeneration $[20,28]$. It has been reported that individuals with MS suffered from spasticity [21] and most of the individuals with MS had gait impairment strongly related to several neurological features, including spasticity [17].

Hypertonicity is a complex symptom including neural and non-neural components [2]. Decreased mobility of the non-neural connective tissue is considered a nonneural component of hypertonicity [2,25]. Spasticity is considered an important neural contributor to muscle hypertonia $[2,25]$. Spasticity is also a common symptom characterized by a velocity-dependent increase in tonic stretch reflexes (muscle tone), and is known as one component of upper motor neuron syndrome [25, 31]. Hypertonic plantar flexor muscles lead to abnormal initial contact with the lateral border of the forefoot and produce an incongruous weight distribution; therefore it disturbs 
the harmonious load transmission [17, 23]. Spasticity also affects normal plantigrade contact by disturbing accommodation to the ground while walking. Plantar flexor hypertonicity is a common symptom in individuals with multiple sclerosis (MS) and affects the walking adaptability of these subjects [17].

Management of spasticity is important for clinicians to improve patients' functionality and walking stability. There are various physiotherapeutic methods to manage spasticity in different neurological conditions such as hydrotherapy, exercise therapy, robotic therapy, etc. $[1,5,8,13,16,18]$. To our knowledge and our clinical observation, myofascial release techniques (MFR) and passive stretching exercises are also widely used in the clinics to decrease the spasticity-related symptoms and spasticity [2].

Myofascial release is a massage technique that focuses on soft tissue that is tight or in spasm [27]. MFR are known as gentle and tolerable techniques and different types of myofascial techniques have been identified in previous studies, such as soft tissue mobilization, trigger point stimulation, etc. [26, 27]. Although there have been some studies investigating the effectiveness of MFR on hypertonus [26], there has been no study investigating the effects of MFR on spasticity in individuals with MS. Also, the effectiveness of passive stretching on spasticity is controversial. Bovend'Eerdt TJ et al.'s [6] systematic review study implied that there was a need for consensus on a paradigm for stretching and future research should address investigating the clinical importance of the short- and long-term effects of stretching techniques on spasticity.

We expected the study to provide evidence whether the myofascial release techniques are effective in the management of spasticity in individuals with MS. Also, we aimed to obtain objective results about the effects of myofascial release and stretching on hind foot loading in individuals with MS.

\section{Material and methods}

The study was planned as a cross-sectional, blinded study.

\section{Participants}

Twenty-three potential participants (46 feet) with multiple sclerosis who were referred to undergo an out-patient neurorehabilitation program at the university physiotherapy and rehabilitation department were screened for the study. Ten participants ( 20 feet; $\mathrm{n}=5$ male, $\mathrm{n}=5$ female; mean age was $39.55 \pm 9.2$ years) who met the following inclusion criteria were recruited for the study: aged within $20-55$ years, had plantar flexor spasticity and an Expanded Disability Status Scale (EDSS) score from 2.0 to 4.0 , able to attend the study on two consecutive days. Exclusion criteria were: had another health problem such as vertebral anomalies, orthopedic problems, rheumatologic problems, recent injury history and passive limitation of lower extremity joints, Botox history in recent 6 months in any extremity, change in medication program in last 2 months; also for women: pregnancy or breastfeeding history in last 3 years, being in the menstrual cycle. Thirteen participants were excluded due to three of them having Botox history, five of them not having spasticity, two of them would not be able to participate on two consecutive days, and three of them need aid to walk. The university's ethical committee approved this study (G017/311; date: 24 Apr 2017).

\section{Interventions}

Following the initial evaluation, participants with multiple sclerosis were given MFR for their plantar flexor muscle group and hamstring distal tendons which connect the crural bones. After the day following the first visit, participants were asked to come again and stretching exercises were applied to the plantar flexor muscle groups after the evaluation.

In our study, MFR was used for releasing the plantar flexor muscles and fascia because it is believed that MFR will not aggravate spasticity and is widely used in clinical practice. MFR was applied regarding the following strategies: the patient lay in the prone position with knee extension. "Therapist 1" stood at the caudal side of the patient and placed the patient's plantar surface at the therapist's femoral region. Achilles, tibialis posterior, peroneus longus and brevis distal tendons, gastrocnemius proximal tendons, and hamstring's distal tendons were mobilized in the first stage. The second stage of MFR comprised deep fascial mobilization of plantar flexor muscle trunks. After that, the skin was also gently mobilized. During MFR, once the spastic muscle relaxation was obtained, the ankle was moved to dorsiflexion gradually. MFR was carried out for 15 minutes for each foot.

The same participants received passive stretching (PS) consisting of 30-minutes stretching and 10-minutes resting periods for each foot on the following day. The patient lay in the supine position with knee extension. The physiotherapist handled the heel and placed the patient's plantar surface at the therapist 1's antebrachial region. Another hand of "physiotherapist 1" was located at the knee to prevent knee flexion while stretching and to augment the effect of passive stretching. After positional preparation, "physiotherapist 1" put forward the ankle to the dorsiflexion and waited at the last point for 30 minutes and then rested for 10 minutes. "Physiotherapist 1 " held the foot at the last position and did not allow any movement of the foot during the resting period. After the resting period, 
"physiotherapist 1 " pushed the ankle to the dorsiflexion direction and held against the resistance for 30 minutes.

\section{Assessments}

Spasticity of the plantar flexor muscles was evaluated using the Modified Ashworth Scale (MAS) in the supine position with the maximal extension of the knee by "physiotherapist 2". MAS is a clinical assessment tool to determine the severity of spasticity. It is scored from 0 to 4 as follows: 0 means no increase in muscle tone; 1 means a slight increase in muscle tone at the end of the range of motion; $1+$ means a slight increase in muscle tone through less than half of the range of motion; 2 means a more marked increase in muscle tone through most of the range of motion; 3 means a considerable increase in muscle tone; and 4 means the joint is rigid [14].

Dynamic pedobarographic analyses $[15,17]$ were used for evaluation of dynamic loading of heels by using a midgait protocol. The pressure platform of the pedobarograph was calibrated by the manufacturer according to standard procedures. Medial and lateral maximum pressure $\left(\mathrm{N} / \mathrm{cm}^{2}\right)$, active contact areas $\left(\mathrm{cm}^{2}\right)$, and contact percentiles $(\%)$ of the heel were recorded. The participants of the present study were asked to walk barefoot at their self-selected speed. Maximum pressure $\left(\mathrm{N} / \mathrm{cm}^{2}\right)$ refers to the greatest loading. Active contact area refers to the greatest area while weight bearing. Contact percentile refers to the maximum contact area with or without weight bearing. Specifications of device: Dimensions were $578 \mathrm{~mm} \times 418 \mathrm{~mm} \times 12 \mathrm{~mm}$; number of sensors was 4096 (arranged in a $64 \times 64$ matrix); sensor dimensions were $7.62 \mathrm{~mm} \times 5.08 \mathrm{~mm}$; active sensor area was $488 \mathrm{~mm} \times 325 \mathrm{~mm}$; sensor technology was resistive; pressure range was $1-127 \mathrm{~N} / \mathrm{cm}^{2}$; data acquisition frequency was $300 \mathrm{~Hz}$; resolution was 8 bits; operating temperature range was $+15^{\circ} \mathrm{C}$ to $+30^{\circ} \mathrm{C}$; storage temperature range was $+0^{\circ} \mathrm{C}$ to $+40{ }^{\circ} \mathrm{C}$; relative humidity was $20 \%$ to $80 \%$ non-condensing.

Assessments were made before and after the MFR and PS were applied. In other words, assessments were performed initially, after MFR application, 24 hours after initial evaluation (before applying passive stretching) and after passive stretching. Dynamic pedobarography was applied by an engineer, spasticity assessment was performed by physiotherapist 2 , and interventions were performed by physiotherapist 1 . The assessors did not know which intervention would be applied on the first day or second day to provide blindness. Also, statistical analyses were performed by a specialist at the university biostatistical department who was blind to the interventions. The flow diagram of the study in shown in Figure 1.

\section{Data analysis}

Histogram and Kolmogorov-Smirnov tests were used to determine whether the parameters were distributed normally or not. According to the test results it was decided to use a nonparametric test. Friedman tests were conducted to test the time-dependent results. Wilcoxon's signed rank test was used to test within-group changes. To compare the effectiveness of MFR and PS the effect size (Cohen d) was calculated. An overall 5\% type-I error level was used to infer statistical significance. Post-hoc power analyses were used to determine the power of the study. The power analysis showed that the power of the study was $>90 \%$ with 0.78 effect size and 10 participants with MS. G*Power software was used to calculate effect size, power of the study and sample size [12].

\section{Results}

According to time-dependent analyses there were differences in maximum pressure of the medial heel of the right $(p=0.01)$ and left foot $(p=0.029)$ and the active contact area of the medial heel of the left foot $(p=0.008)$. Pairwise analyses showed that there was a difference between the first and the second evaluations for the maximum pressure of the medial heel of the right foot $(p=0.008 ; d=0.78)$; in other words the maximum pressure of the medial heel of the right foot was increased. There were differences between the first and second evaluation ( $\mathrm{p}=0.008 ; \mathrm{d}=1.2)$, and the second and third evaluations $(\mathrm{p}=0.012 ; \mathrm{d}=0.105)$ for the maximum pressure of the medial heel of the left foot; maximum pressure of the medial heel increased after MFR application and wash out until the second day. There were differences between the first and second evaluations $(\mathrm{p}=0.015 ; \mathrm{d}=1.38)$ and second and third evaluations $(p=0.017 ; d=0.031)$ for the active contact area of the medial heel of the left foot; the active contact area was increased after MFR and decreased again until the second day. The results clearly showed that after MFR the maximum pressure of the medial heel and the active contact area were increased and there was a carryover effect in the maximum pressure of the right foot. The results are summarized in Table 1 . Analysis of 20 feet showed that there were no differences in MAS $(p>0.05)$ in time-dependent analyses (Table 2).

\section{Discussion}

This study showed that the myofascial release techniques decreased spasticity by decreasing the fascia resistance which strongly-related with muscle resistance. Therefore, it can be said that the myofascial release techniques are effective methods for management in patients with multiple sclerosis who had spasticity in plantar flexor muscles in the short term and there was a carryover effect in favor of the myofascial release techniques. While 


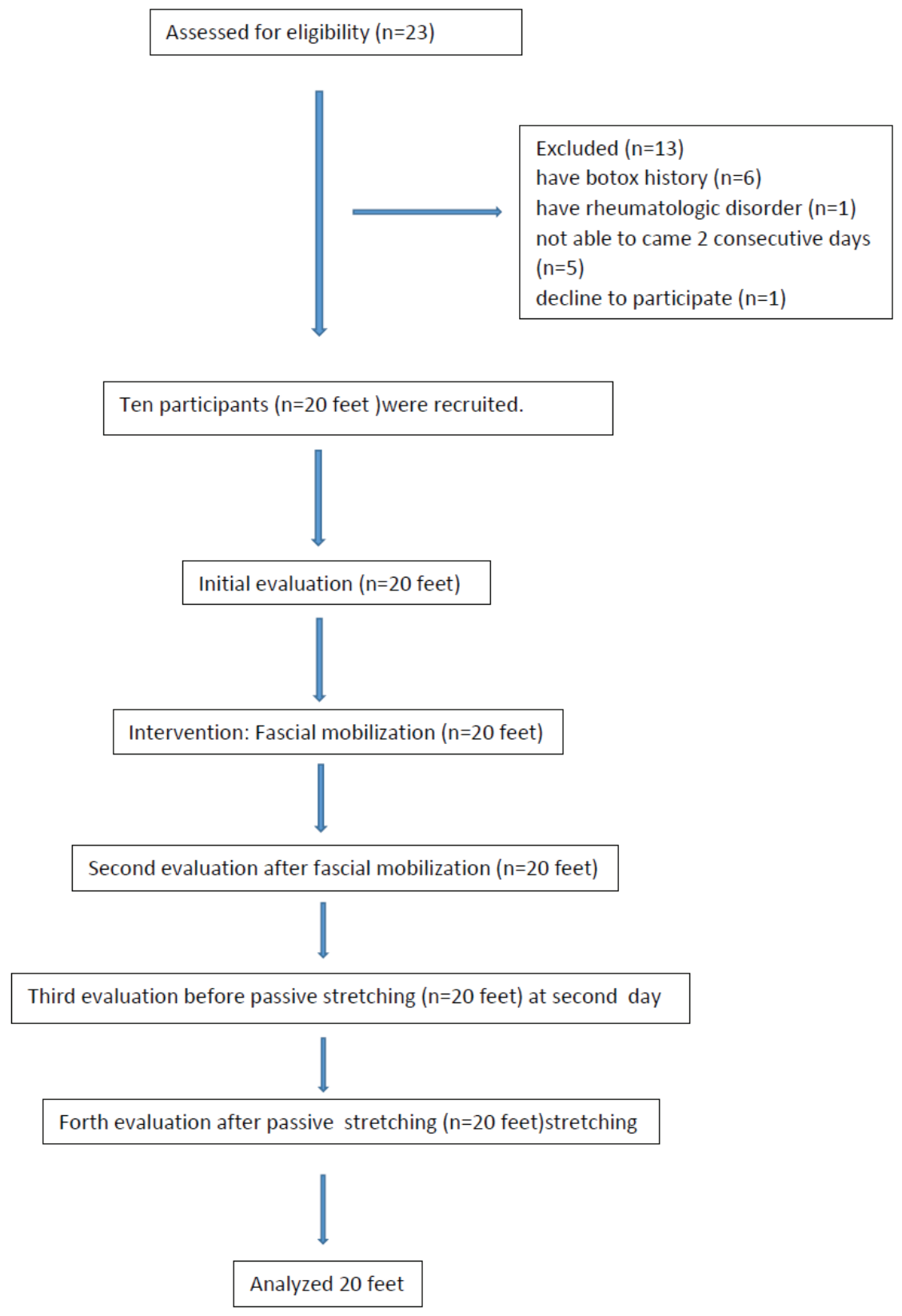

Fig. 1. The flow chart of the study

the spasticity values were not changed, the plantigrade contact ability of the hind foot improved after the MFR application. There was no favorable effect of the passive stretching.

Previous studies carried out in children with spastic cerebral palsy implied that myofascial release was useful in the management of spasticity [4, 10, 19, 29]. Kumar et al. [19] investigated the effect of myofascial release on spastic muscles. Children with spastic diplegia were given conventional physiotherapy plus myofascial release or only conventional physiotherapy for 4 weeks. The study concluded that myofascial release along with conventional treatment reduces spasticity in calf, hamstring, and adductors of hip muscles [19]. A 2014 study also showed that stretching can be used along with myofascial release in reducing spasticity in patients with spastic cerebral palsy rather than using stretching alone [4]. In the Diwan SJ et al. study, children with spastic cerebral palsy received myofascial release for the anterior chest wall - diaphragm, rectus abdominis, pectoralis major, intercostal muscles - given for 5 days a week for a month. Chest measurements were taken at three levels at the axilla, 3 fingers below the xiphoid process, and subcostal region. The results of this study showed that the chest expansion increased significantly [10]. In our study, while MFR did not decrease spasticity, there were improvements of medial heel pressure distribution and active contact area while walking. It has been previously 


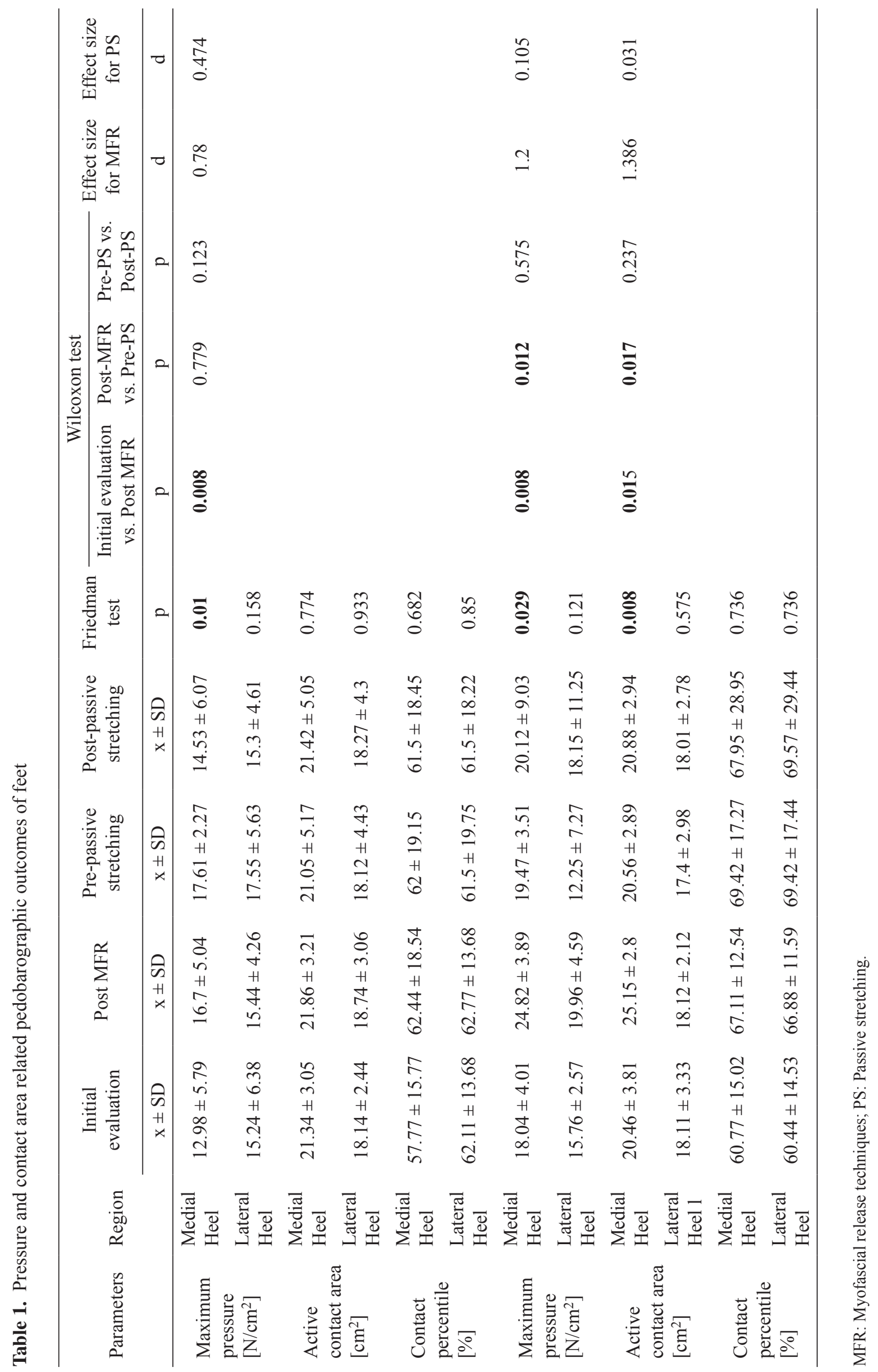


Table 2. Spasticity-related results

\begin{tabular}{|c|c|c|c|c|c|}
\hline & & Mean & Minimum & Maximum & Friedman test $p$ \\
\hline \multirow{4}{*}{ Right feet } & Initially & 2 & 1 & 4 & \multirow{4}{*}{0.673} \\
\hline & Post-MFR & 1.88 & 1 & 4 & \\
\hline & Pre-Stretching & 1.77 & 1 & 4 & \\
\hline & Post-Stretching & 2 & 1 & 4 & \\
\hline \multirow{4}{*}{ Left feet } & Initially & 1.77 & 1 & 4 & \multirow{4}{*}{0.988} \\
\hline & Post-MFR & 1.77 & 1 & 4 & \\
\hline & Pre-Stretching & 1.77 & 1 & 4 & \\
\hline & Post-Stretching & 1.77 & 1 & 4 & \\
\hline
\end{tabular}

Note that: "1+" score accepted as 2 for calculation of statistical analyses; MFR: myofascial release techniques.

shown that spasticity was not directly related to function and that improvements in motor indicators of spasticity did not always consider gains in the functional use of the extremity [9, 25]. Another possible explanation of our results is that the Modified Ashworth Scale may not be sensitive for detecting small changes in spasticity. We believe that the MFR may decrease the muscle resistance, which is independent from velocity, by affecting the non-neural mechanism of spasticity $[4,26]$.

The carryover effect of MFR was interesting. We cannot explain or discuss these results with previous studies because there is no research investigating the carryover effect of MFR. However, according to the authors of the present study, the MFR could have stimulated the proprioceptive system because it was applied to various tissues. It is believed that passive stretching is stimulated in the autogenic inhibition pattern via the Golgi reflex [30, 31]. It is known that the Golgi response is not permanent [30, 31]. On the other hand, MFR could stimulate the other tissue receptors, such as touch, compression, strain, etc., receptors. Multiple stimulants via MFR techniques may have had a releasing effect on the hypertonic activity of the muscles [4, 29]. Also, the carryover effect of MFR may have resulted from such massage techniques' macrocirculatory outcomes on the tissue release [24]. Nonetheless, these opinions were only claims of the authors; there were no evidence-based results which support these explications. However, these results suggest that MFR can be applied frequently in the clinic. If MFR is performed at a certain frequency, it may result in the aggregation and enhancement of the therapeutic effect of the technique in patients with multiple sclerosis.

There is a controversy in studies that investigate the effectiveness of stretching on spasticity. While some studies revealed that there was no improvement after application of passive stretching, others showed beneficial effects of passive stretching $[3,6,7,22,30]$. The passive stretching applied in the current study was not effective in decreasing the hypertonicity and improving the foot pressure distribution in the short term in patients with multiple sclerosis, but it is still necessary to investigate the long-term effects of passive stretching. Different styles of stretching exercises can be researched in the same and/or different population to reach a consensus on the effectiveness of passive stretching in the management of spasticity in individuals with MS.

\section{Limitations}

There were some limitations of the study. The study was not planned as a randomized controlled trial. Another limitation was that there was no control group with no intervention. We tried to eliminate the effect of this limitation by using the effect size. In other words, the results were only interpreted with the effect size, a kind of statistical method providing knowledge of the intervention effectiveness. Also, the study could have been carried out with a larger number of patients.

\section{Conclusion}

This study showed that myofascial release techniques decreased the spasticity by mobilizing the stiff fascia and decreasing the thickness of the plantar flexor muscles are effective methods for management in patients with multiple sclerosis who had spasticity in the plantar flexor muscles in the short term and there was a carryover effect in favor of the myofascial release techniques. While the spasticity values were not changed, the plantigrade contact ability of the hind foot improved after the MFR application. It can be used as an adjunct to treatment. Whether using the myofascial release techniques in the long term has any additional benefits requires further investigation. Also, there was no favorable effect of passive stretching. 
Conflict of interest: Authors state no conflict of interest.

\section{References}

1. Albert T., Yelnik A. (2003) Physiotherapy for spasticity. Neurochirurgie, (49): 239-246.

2. Armutlu K. (2010) Spasticity \& its Management with Physical Therapy Applications. Nova Science Publishers Inc.

3. Bakheit A.M.O., Maynard V., Shaw S. (2005) The effects of isotonic and isokinetic muscle stretch on the excitability of the spinal alpha motor neurones in patients with muscle spasticity. Eur. J. Neurol., 12(9): 719-724.

4. Bhalara A., Talsaniya D. (2014) Short Term Effect of Myofascial Release on Calf Muscle Spasticity in Spastic Cerebral Palsy Patients. Int. J. Health Sci. Res., 4(9): 188-194.

5. Boyd R.N., Ada L. (2001) Physiotherapy management of spasticity. Upper motor neurone syndrome and spasticity: clinical management and neurophysiology. Cambridge University Press, 79-81.

6. Bovend'Eerdt T.J., Newman M., Barker K., Dawes H., Minelli C., Wade D.T. (2008) The effects of stretching in spasticity: a systematic review. Arch. Phys. Med. Rehabil., 89(7): 1395-1406.

7. Bressel E., McNair P.J. (2002) The effect of prolonged static and cyclic stretching on ankle joint stiffness, torque relaxation, and gait in people with stroke. Phys. Ther., 82(9): 880-887.

8. Camerota F., Celletti C., Di Sipio E., De Fino C., Simbolotti C., Germanotta M., Mirabella M., Padua L., Nociti V. (2017) Focal muscle vibration, an effective rehabilitative approach in severe gait impairment due to multiple sclerosis. J. Neurol. Sci., 372: 33-39.

9. de Vlugt E., de Groot J.H., van der Heijden-Maessen H.C., Wielheesen D.H., van Wijlen-Hempel R.M.S., Arendzen J.H., Meskers C.G. (2013) Differentiation between nonneural and neural contributors to ankle joint stiffness in cerebral palsy. J. Neuroeng. Rehabil., 10(1): 81.

10. Diwan S.J., Bansal A.B., Chovatiya H., Kotak D., Vyas N. (2014) Effect of anterior chest wall myofascial release on thoracic expansion in children with spastic cerebral palsy. Int. J. Contemp. Pediatr., 1(2): 94-99.

11. Evangelou N., Paine S.M., Tallantyre E.C. (2018) The Neuropathology of Progressive Multiple Sclerosis. In Progressive Multiple Sclerosis. Springer, 49-69.

12. Faul F., Erdfelder E., Lang A.G., Buchner A. (2007) G* Power 3: A flexible statistical power analysis program for the social, behavioral, and biomedical sciences. Behav. Res. Methods, 39(2): 175-191.

13. Fazekas G., Horvath M., Troznai T., Toth A. (2007) Robot-mediated upper limb physiotherapy for patients with spastic hemiparesis: a preliminary study. J. Rehabil. Med., 39(7): 580-582.
14. Ghotbi N., Ansari N.N., Naghdi S., Hasson S. (2011) Measurement of lower-limb muscle spasticity: intrarater reliability of Modified Modified Ashworth Scale. J. Rehabil. Res. Dev., 48(1): 83-8.

15. Hughes J. (1993) The clinical use of pedobarography. Acta Orthop. Belg., 59(1): 10-16.

16. Kaya Kara O., Livanelioglu A., Yardımcı B.N., Soylu A.R. (2019) The Effects of Functional Progressive Strength and Power Training in Children With Unilateral Cerebral Palsy. Pediatr. Phys. Ther., 31(3): 286-295.

17. Keklicek H., Cetin B., Salci Y., Balkan A.F., Altinkaynak U., Armutlu K. (2018) Investigating the dynamic plantar pressure distribution and loading pattern in subjects with multiple sclerosis. Mult. Scler. Relat. Disord., 20: 186-191.

18. Kesiktas N., Paker N., Erdogan N., Gülsen G., Biçki D., Yilmaz H. (2004) The use of hydrotherapy for the management of spasticity. Neurorehabil. Neural. Repair, 18(4): 268-273.

19. Kumar C., Vaidya S.N. (2014) Effectiveness of Myofascial Release on Spasticity and Lower Extremity Function in Diplegic Cerebral Palsy: Randomized Controlled Trial. Int. J. Phys. Med. Rehabil., 3: 1.

20. Lassmann H. (2018) Multiple sclerosis pathology. Cold Spring Harb. Perspect. Med., 1-8: 37.

21. Lucchinetti C., Brück W., Parisi J., Scheithauer B., Rodriguez M., Lassmann H. (2000) Heterogeneity of multiple sclerosis lesions: implications for the pathogenesis of demyelination. Ann. Neurol., 47: 707-717.

22. Maynard V., Bakheit A.M., Shaw S. (2005) Comparison of the impact of a single session of isokinetic or isotonic muscle stretch on gait in patients with spastic hemiparesis. Clin. Rehab., 19(2): 146-154.

23. Molteni F., Bertoni M., Biong M.C., Biong L.M., Biong M.R. (2002) Equinovarus foot in upper motor neuron syndrome. Phys. Med. Rehabil., 16(2): 263.

24. Moyer C.A., Rounds J., Hannum J.W. (2004) A metaanalysis of massage therapy research. Psychol. Bull., 130(1): 3 .

25. Mukherjee A., Chakravarty A. (2010) Spasticity mechanisms - for the clinician. Front. Neurol., 1:149.

26. Paolini J. (2009) Review of myofascial release as an effective massage therapy technique. Athl. Ther. Today, 14(5): 30-34.

27. Sefton J. (2004) Alternative and complementary concepts. myofascial release for athletic trainers, part I: theory and session guidelines. Athl. Ther. Today, 9(1): 48-49.

28. Trapp B.D., Nave K.A. (2008) Multiple sclerosis: an immune or neurodegenerative disorder? Annu. Rev. Neurosci., 31: 247-269.

29. Whisler S.L., Lang D.M. Armstrong M., Vickers J., Qualls C., Feldman J.S. (2012) Effect of myofascial release and other advanced myofascial therapies on children with cerebral palsy: six case reports. Explore, 8: 199-205. 
30. Yeh C.Y., Tsai K.H., Chen J.J. (2005) Effects of prolonged muscle stretching with constant torque or constant angle on hypertonic calf muscles. Arch. Phys. Med. Rehabil., 86(2): 235-241.

31. Young R.R., Delwaide P.J. (1981) Spasticity. N. Engl. J. Med., 304(1): 28-33.

\section{Received 28.03.2019}

Accepted 11.02.2020

(C) University of Physical Education, Warsaw, Poland 